\title{
Anisotropic Improved Gauge Actions: - Perturbative and Numerical Studies -
}

\author{
S. Sakai, ${ }^{\mathrm{a}}$, A. Nakamura, ${ }^{\mathrm{b}}$, and T.Saito, ${ }^{\mathrm{c}}$ \\ ${ }^{a}$ Faculty of Education, Yamagata University \\ ${ }^{\mathrm{b}}$ Research Center for Information Science and Education, Hiroshima University \\ ${ }^{c}$ Department of Physics, Hiroshima University
}

The $\Lambda$ parameter on the anisotropic lattice, the spatial and temperature coupling constant $g_{\sigma}, g_{\tau}$ and their derivative with respaect to the the anisotropy parameter $\xi$ are studied perturbatively for the class of improved actions, which cover tree level Symanzik's, Iwasaki's and QCDTARO's improved actions. The $\eta\left(=g_{\tau} / g_{\sigma}\right)$ becomes less than 1 for Iwasaki's and QCDTARO's action, which is confirmed nonperturbatively by numerical simulations. Derivatives of the coupling constants with respect to the anisotropy parameter, $\partial g_{\tau} / \partial \xi$ and $\partial g_{\sigma} / \partial \xi$, change sign for those improved actions.

\section{INTRODUCTION}

The basic parameters of anisotropic lattices with improved action are important information for numerical simulations. In this work, we mainly focus on perturbative calculations of these parameters, but some of them are confirmed by numerical simulations.

The improved actions we study in this work consist of terms

$$
S_{\mu \nu}=\alpha_{0} \cdot L(1 \times 1)_{\mu \nu}+\alpha_{1} \cdot L(1 \times 2)_{\mu \nu}
$$

where $L(1 \times 1)$ and $L(1 \times 2)$ represent plaquette and 6 -link rectangular loops respectively, and $\alpha_{0}$ and $\alpha_{1}$ satisfies $\alpha_{0}+8 \cdot \alpha_{1}=1$. The improved actions cover i) tree level Symanzik's action $\left(\alpha_{1}=\right.$ $-1 / 12)$ 1], ii) Iwasaki's action $\left(\alpha_{1}=-0.331\right)$ 2], and iii) QCDTARO's action $\left(\alpha_{1}=-1.409\right)$ 3] etc.

For these classes of improved actions the anisotropic lattice is formulated in the same way as in the case of standard plaquette action [4. We introduce the coupling constant and lattice spacing in space direction $g_{\sigma}, a_{\sigma}$ and those in temperature direction $g_{\tau}, a_{\tau}$. With these parameters the action on the anisotropic lattice is written as,

$$
S=\beta_{\sigma} \cdot S_{i j}+\beta_{\tau} \cdot S_{4 i}
$$

where $\beta_{\sigma}=g_{\sigma}^{-2} \xi_{R}^{-1}, \beta_{\tau}=g_{\tau}^{-2} \xi_{R}$ and $\xi_{R}=a_{\sigma} / a_{\tau}$.

In this study, $\Lambda$ parameter and the ratio $\eta=$ $g_{\tau} / g_{\sigma}$ are calculated and the derivatives of the coupling constants with respect to the anisotropy parameter, $\partial g_{\sigma}^{-2} / \partial \xi_{R}, \partial g_{\tau}^{-2} / \partial \xi_{R}$ will be discussed.

\section{PERTURBATIVE CALCULATIONS}

In weak coupling expansions, the relation between the couping constants on the anisotropic lattice and those on the isotropic lattice are obtained as follows[4]. We calculate the coefficients of $F_{i j}^{n} F_{i j}^{n}$ and $F_{i 4}^{n} F_{i 4}^{n}$ in the action in one loop order.

$$
\begin{aligned}
& S_{E f f}\left(\xi_{R}\right) \\
& \quad=\frac{1}{4}\left(g_{\sigma}^{-2}-C_{\sigma}\left(\xi_{R}\right)\right) \cdot F_{i j}^{n} F_{i j}^{n} \cdot a_{\sigma}^{3} a_{\tau} \\
& \quad+\frac{1}{4}\left(g_{\tau}^{-2}-C_{\tau}\left(\xi_{R}\right)\right) \cdot F_{4 i}^{n} F_{4 i}^{n} \cdot a_{\sigma}^{3} a_{\tau}
\end{aligned}
$$

The same calculation is done on the isotropic lattice. We require that the effective action is independent of the method of regularization. Then the relations are derived.

$g_{\sigma}^{-2}\left(\xi_{R}\right)=g(1)^{-2}+\left(C_{\sigma}\left(\xi_{R}\right)-C_{\sigma}(1)\right)+O\left(g(1)^{2}\right)$ $g_{\tau}^{-2}\left(\xi_{R}\right)=g(1)^{-2}+\left(C_{\tau}\left(\xi_{R}\right)-C_{\tau}(1)\right)+O\left(g(1)^{2}\right)$ On an isotropic lattice $\left(\xi_{R}=1\right)$, gauge invariance means $C_{\sigma}(1)=C_{\tau}(1)$. 
For the calculation of $C_{\sigma}$ and $C_{\tau}$, we employ the background field method [0] used by Iwasaki and Sakai[6] in the calculation of the $\Lambda$ parameters of improved actions on the isotropic lattice. The details of the method will be reported in the forthcoming publications.

The $C_{\tau}\left(\xi_{R}\right)$ and $C_{\sigma}\left(\xi_{R}\right)$ have infrared divergences, but in the difference they are cancelled. The cancellation of the divergence is a delicate problem. The following method is used.

$$
\begin{aligned}
& \Delta C_{\sigma}\left(\xi_{R}\right)=C_{\sigma}^{\operatorname{Imp}}\left(\xi_{R}\right)-C_{\sigma}^{\operatorname{Imp}}(1) \\
& \quad=\left(C_{\sigma}^{\operatorname{Imp}}\left(\xi_{R}\right)-C_{\sigma}^{\text {Stand }}\left(\xi_{R}\right)\right) \\
& \quad+\left(C_{\sigma}^{\text {Stand }}\left(\xi_{R}\right)-C_{\sigma}^{\text {Stand }}(1)\right) \\
& \quad+\left(C_{\sigma}^{\text {Stand }}(1)-C_{\sigma}^{\text {Imp }}(1)\right)
\end{aligned}
$$

In the first and third terms, the infrared divergence is canceled exactly for improved and standard actions. The second term is already calculated by Karsch [4] where the regularization of the infrared divergence could be done by an analytic integration of the fourth component of the loop momentum.

With these $C_{\sigma}\left(\xi_{R}\right), C_{\tau}\left(\xi_{R}\right)$ functions, $\Lambda$ and $\eta$ are expressed as follows.

$$
\begin{gathered}
\Lambda\left(\xi_{R}\right) / \Lambda(1)=\exp \left(-\frac{\Delta C_{\sigma}\left(\xi_{R}\right)+\Delta C_{\tau}(\tau)}{4 b_{0}}\right) \\
\eta=\frac{g_{\tau}}{g_{\sigma}}=1+N \cdot\left(C_{\sigma}-C_{\tau}\right) / \beta+O\left(g^{4}\right)
\end{gathered}
$$

The ratios $\Lambda\left(\xi_{R}\right) / \Lambda$ are shown in Fig.1. Except for the QCDTARO's action, they have similar $\xi_{R}$ dependences. The more interesting differences are seen in the ratio $\eta$. In Fig.2, we show $C_{\sigma}\left(\xi_{R}\right)-C_{\tau}\left(\xi_{R}\right)$, which determines $\eta$. It is found that as $\alpha_{1}$ decreases $C_{\sigma}\left(\xi_{R}\right)-C_{\tau}\left(\xi_{R}\right)$ changes sign. Namely for Iwasaki's and QCDTARO's improved actions, $\eta<1$, and at $\alpha_{1} \sim-0.160$, $C_{\sigma}\left(\xi_{R}\right)-C_{\tau}\left(\xi_{R}\right) \sim 0$ for all $\xi_{R}$. This result is very interesting and is not what we have expected. Then in the next section, we calculate the parameter $\eta$ nonperturbatively and confirm the perturbative results.

\section{NONPERTURBATIVE STUDY OF $\eta$}

Nonperturbatively, the parameter $\eta$ is calculated by the relation $\eta=\xi_{R} / \xi_{B}[7][$ [ [ [9], where

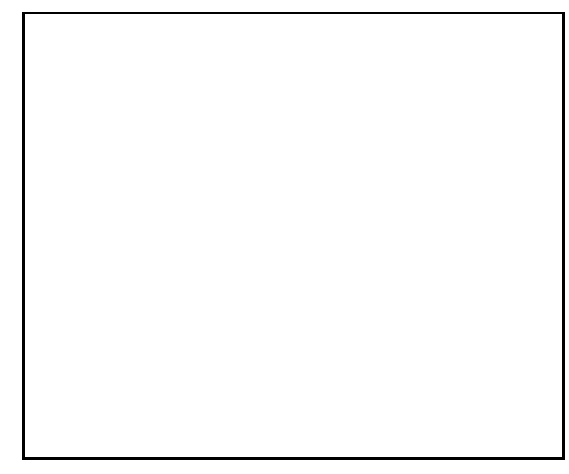

Figure 1. The ratio $\Lambda\left(\xi_{R}\right) / \Lambda(1)$ for improved and standard actions

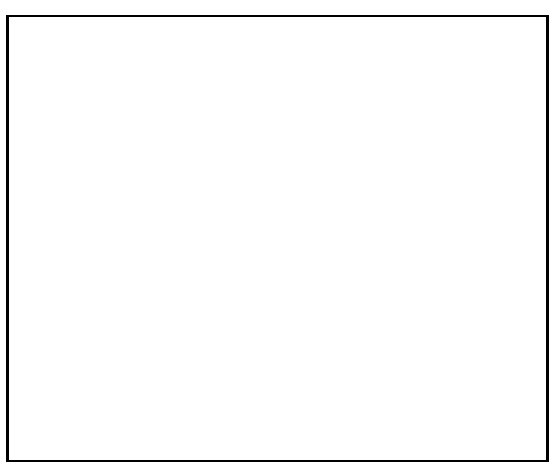

Figure 2. $C_{\sigma}\left(\xi_{R}\right)-C_{\tau}\left(\xi_{R}\right)$ as a function of $\alpha_{1}$

$\xi_{B}$ is a bare the anisotropy parameter which appears in the action,

$$
S=\frac{2 N}{g^{2}}\left(\frac{1}{\xi_{B}} S_{i j}+\xi_{B} S_{i 4}\right)
$$

while the renormalized the anisotropy parameter is defined by $\xi_{R}=a_{\sigma} / a_{\tau}$. For the probe of the scale in the space and temperature direction, we use the lattice potential in these directions which is defined by,

$$
V_{s t}\left(\xi_{B}, l, t\right)=\ln \left(\frac{W_{s t}(l, t)}{W_{s t}(l+1, t)}\right)
$$


similarly for the potential in the space direction. Here we fix $\xi_{R}=2$, and calculate the rate for a few $\xi_{B}$

$$
R\left(\xi_{B}, l, r\right)=\frac{V_{s s}\left(\xi_{B}, l, r\right)}{V_{s t}\left(\xi_{B}, l, \xi_{R} t\right)}
$$

Then we search for the point $R=1$ by interpolating with respect to $\xi_{B}[7][8]$.

The simulations are done on the $12^{3} \times 24$ lattice, in the large $\beta$ region. The results are shown in Fig.3, together with those of perturbative calculations.

It is found that the agreement of both results

Figure 3. Perturbative and Nonperturbative results for $\eta\left(\xi_{R}=2, \beta\right)$

is good. So the $\alpha_{1}$ dependences of the $\eta$ ratio is also confirmed by the numerical simulation, a very exciting result for improved actions.

\section{ESTIMATION OF $\frac{\partial g_{\sigma}^{-2}}{\partial \xi_{R}}$ AND $\frac{\partial g_{\tau}^{-2}}{\partial \xi_{R}}$}

The exact calculation of these derivatives are not carried out yet. However we could estimate them from the slope of the $C_{\sigma}\left(\xi_{R}\right)$ and $C_{\tau}\left(\xi_{R}\right)$ at $\xi_{R}=1$ and $\xi_{R}=1.01$. The results are shown in Fig.4. They should satisfy following sum rule俚,

$$
\frac{\partial C_{\sigma}}{\partial \xi_{R}}+\left.\frac{\partial C_{\tau}}{\partial \xi_{R}}\right|_{\xi_{R}=1}=\frac{11 \times N}{48 \pi^{2}}
$$

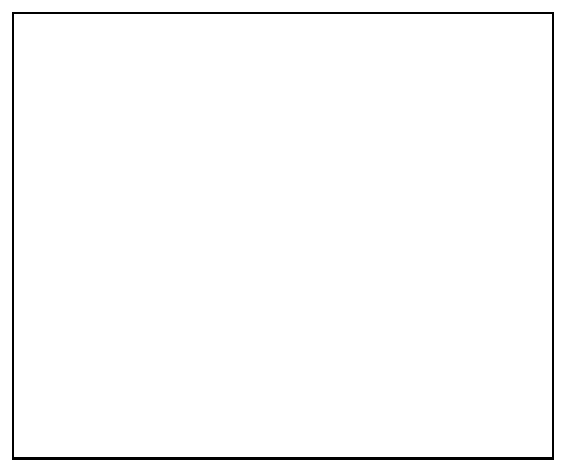

Figure 4. $\partial g_{\sigma}^{-2} / \partial \xi_{R}$, and $\partial g_{\tau}^{-2} / \partial \xi_{R}$ from numerical estimation

The saturation of the sum rule is good.

Notice that the derivatives change sign as $\alpha_{1}$ decreases. These coefficients play important role in the calculation of internal energy and pressure. As the sign of these coefficients changes for the improved actions, it may be interesting to calculate thermodynamic quantities with these improved actions.

In conclusion, the study of anisotropic lattice with improved action shows very interesting properties as shown in Figs.1,2,3,4. The study of finite temperature physics with these improved action seems to be very exciting.

\section{REFERENCES}

1. K. Symanzik, Nucl. Phys. B226(1983), 187

2. Y. Iwasaki, Nucl. Phys. B258(1985), 141; Univ. of Tsukuba preprint UTHEP-118(1983)

3. QCD-TARO Collaboration, Ph. de Forcrand et al. hep-lat/9806008

4. F.Karsch, Nucl. Phys.B205[FS5](1982), 285

5. R.Dashen and D.Gross, Phys. Rev. D23(1981),2340

6. Y. Iwasaki and S. Sakai, Nucl. Phys. B248(1984), 441

7. T.R.Klassen hep-lat/9803010

8. G.Burgers et al., Nucl. Phys. B204, 587, 1988

9. J. Engels, F. Karsch, T. Scheideler, Nucl.Phys.B(Proc Suppl)63A-C(1998),48 


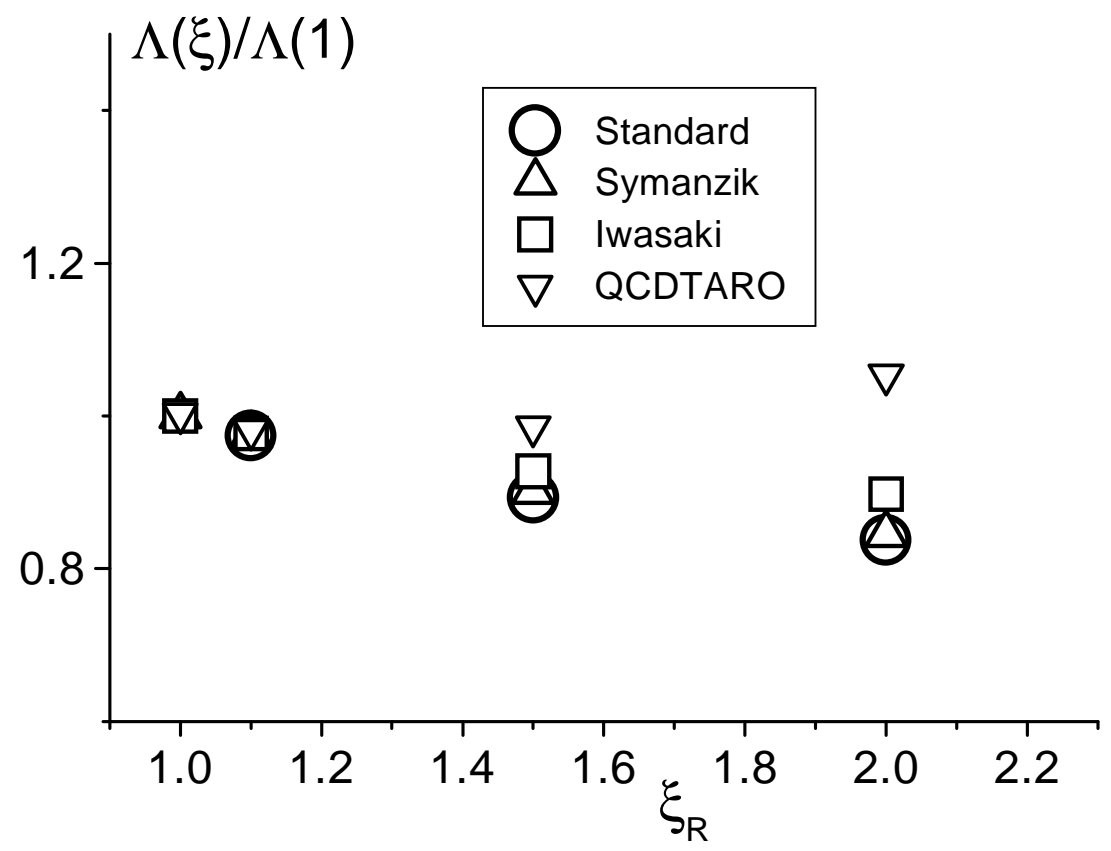

Fig.1 


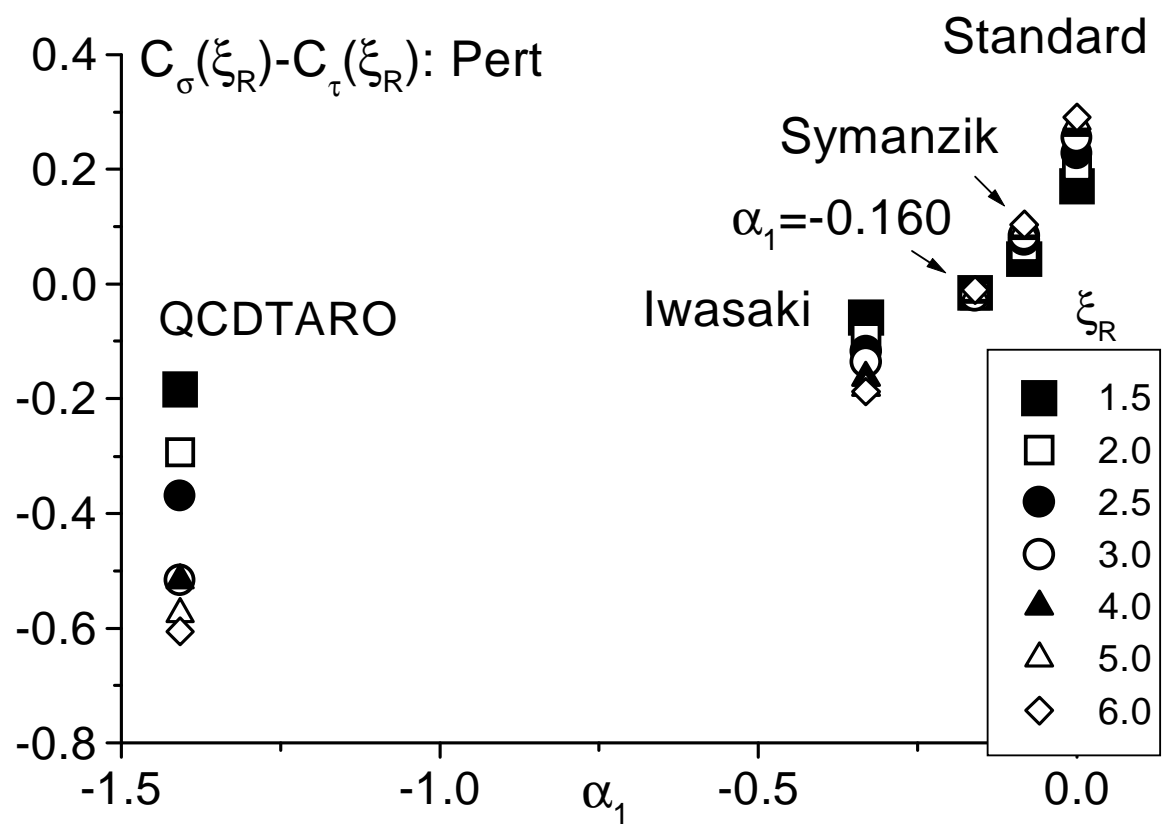

Fig.2 


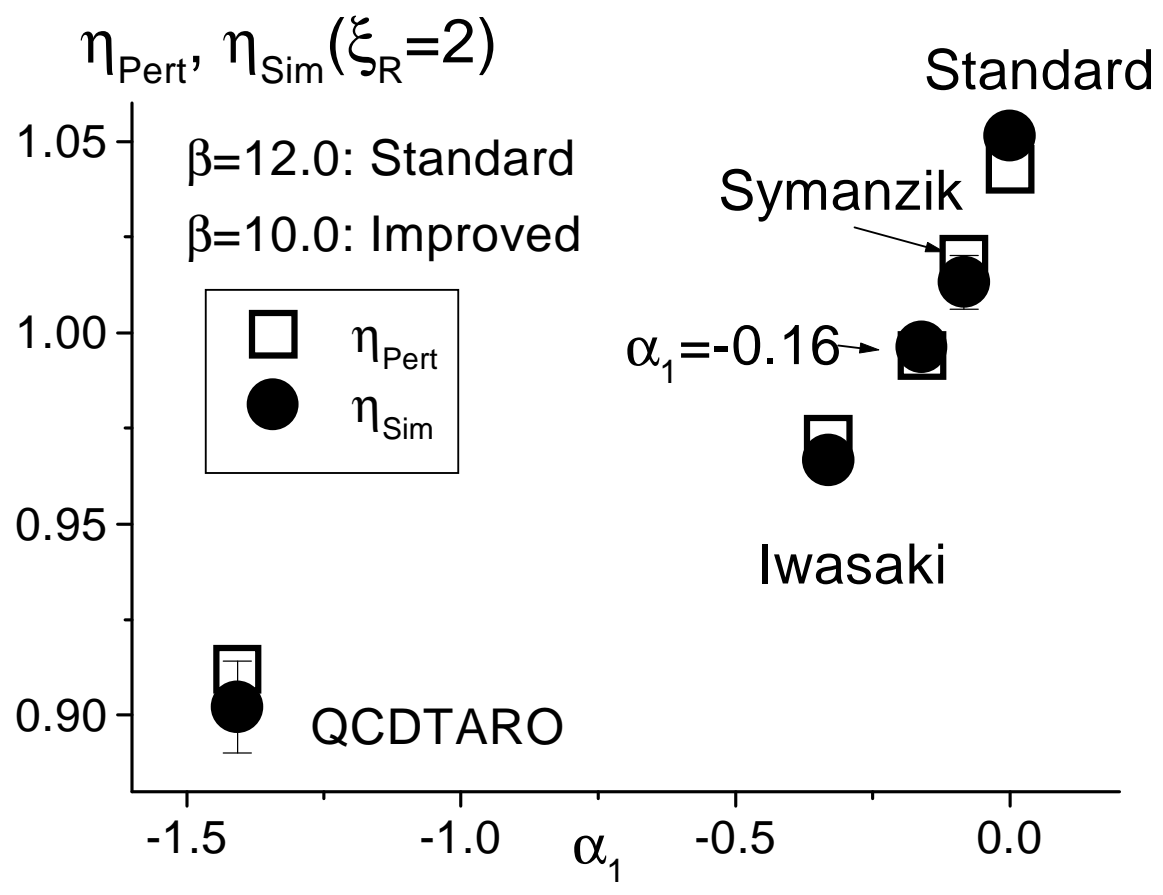

Fig.3 


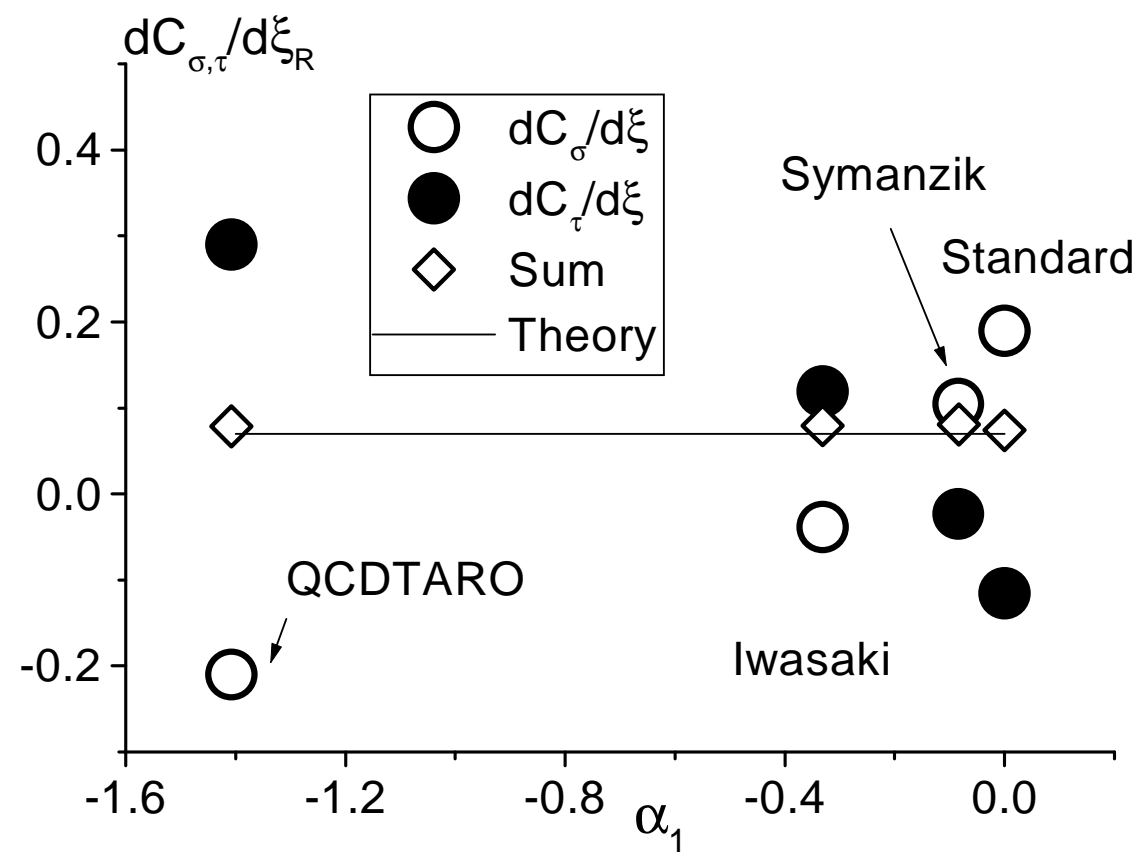

Fig.4 